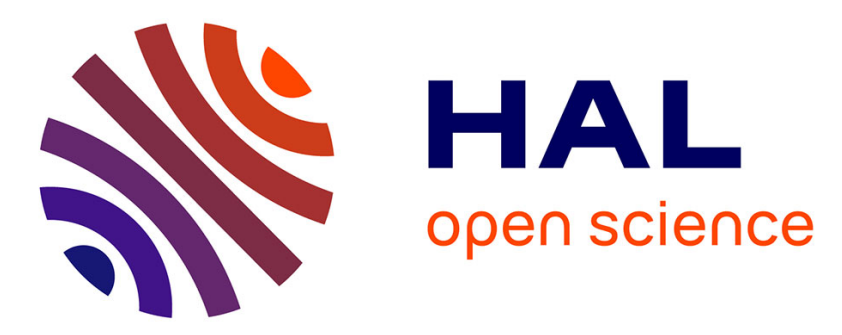

\title{
Classification of high resolution urban satellite Images combining SVM and Graph Cuts
}

\author{
A. Bekkari, S. Idbraim, K. Housni, D. Mammass, Youssef Chahir
}

\section{To cite this version:}

A. Bekkari, S. Idbraim, K. Housni, D. Mammass, Youssef Chahir. Classification of high resolution urban satellite Images combining SVM and Graph Cuts. 2010 5th International Symposium On I/V Communications and Mobile Network (ISVC), Sep 2010, Rabat, France. 10.1109/ISVC.2010.5656433 . hal-01882847

\section{HAL Id: hal-01882847 https://hal.science/hal-01882847}

Submitted on 27 Sep 2018

HAL is a multi-disciplinary open access archive for the deposit and dissemination of scientific research documents, whether they are published or not. The documents may come from teaching and research institutions in France or abroad, or from public or private research centers.
L'archive ouverte pluridisciplinaire HAL, est destinée au dépôt et à la diffusion de documents scientifiques de niveau recherche, publiés ou non, émanant des établissements d'enseignement et de recherche français ou étrangers, des laboratoires publics ou privés. 


\section{Classification of high resolution urban satellite Images combining SVM and Graph Cuts}

\author{
A. Bekkari ${ }^{1}$, S. Idbraim $^{2}$, K. Housni ${ }^{3}$ and D. Mammass ${ }^{4}$ \\ IRF - SIC laboratory, \\ Faculty of sciences \\ Agadir -Morocco \\ a_bekkari@yahoo.fr ${ }^{1}$; soufianeidbraim@yahoo.fr ${ }^{2}$; \\ khalidhousni@yahoo.fr ${ }^{3}$; mammass@univ-ibnzohr.ac.ma ${ }^{4}$
}

\author{
Y. Chahir \\ GREYC-UMR CNRS 6072, \\ University of Caen \\ Caen -France \\ chahir@info.unicaen.fr
}

\begin{abstract}
The classification of remotely sensed images knows a large progress seen the availability of images of different resolutions as well as the abundance of the techniques of classification. Moreover a number of works showed promising results by the fusion of spatial and spectral information. For this purpose we propose a methodology allowing to combine this two information to refine an SVM classification, The approach uses graph cuts to improve the SVM algorithm, as graph cuts introduce spatial domain information of the image that is lacking in the SVM.

The proposed approach is tested on common scenes of urban imagery. The experimental results show satisfactory values and are very promising.
\end{abstract}

Keywords - Graph Cuts; Satellite image; Space and spectral information; $S V M$.

\section{INTRODUCTION}

With the commercial emergence of the optical satellite images of sub-metric resolution (Ikonos, Quickbird) the realization as well as the regular update of numerical charts with large scales becomes accessible and increasingly frequent. The classification of such images is similar to that of other types of images, it follows the same principle, and it is a method of analysis of data that aims to separate the image into several classes of interest, in order to gather the data in homogeneous subsets, which show common characteristics. It aims to assign to each image's pixel a label what it represents in a scene (a zone of vegetation, a water zone, etc) [1].

Several techniques exist, in this work, we use SVM. This method is actually no more than an algorithm of training and classification. It is about a problem of optimization that makes possible the separation of a field into two or several classes. The problem consists in transforming the space in which one works in another space known as large-sized and inside of which we will establish a separation in various classes. The passage from one space to another is carried out according to some mathematical transformations that use the nonlinear functions, called kernel functions.

The proposed method consists in combining space and spectral information to obtain a better classification, we start with a spectral classification with SVM. Then, we apply the Graph Cuts approach which takes in account the space dimensions of the classification. Experimental results are provided and comparisons with morphological approach applied to the spectral classification are made to illustrate that the method is able to find better class.

This paper is organized as follows. In the second section, we give outlines on the used classifier: Support Vector Machines (SVM). In the section 3, the used Graph Cuts approach is described. In Section 4, the results are presented with numerical evaluation. Finally, conclusions are given in Section 5.

\section{SPECTRAL CLASSIFICATION WITH SVM}

The support vectors machines (SVM) [2]-[4] introduced by Vapnik is a technique of construction of optimal hyperplanes separating $N$ classes and which provides a continuous function of discrimination. Data are projected in a deployed space of large dimension (space of Hilbert) in order to define a linear separation in this space, before returning back to the initial space. The choice of separation: hyperplane which classifies the data correctly (when it is possible) and which is "as far as possible from all the examples" [5] as shown in Fig. 1.

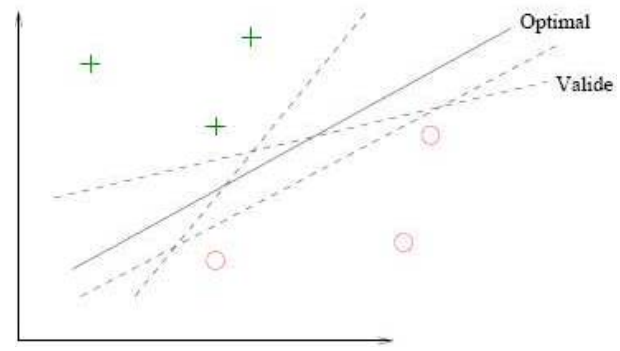

Figure 1. The optimal hyperplane separating two classes

This separation is concretized by the maximization of the margin (a point distance to the plan) given by :

$$
d(\boldsymbol{x})=\frac{|\boldsymbol{w} \cdot \boldsymbol{x}+b|}{\|\boldsymbol{w}\|}
$$

What amounts minimizing $\|\boldsymbol{w}\|$ with constraints, so we get the primal problem of minimization with constraints 


$$
\left\{\begin{array}{l}
\min \frac{1}{2}\|\boldsymbol{w}\|^{2} \\
\forall i, y_{i}\left(\boldsymbol{w} \cdot \boldsymbol{x}_{i}+b\right) \geq 1
\end{array}\right.
$$

We consider the dual problem by introducing the multipliers of Lagrange for each constraint. Here for example there is a constraint of learning that gives us a problem of quadratic programming of dimension $N$ (the number of examples). With the Hessian Matrix: $\left(x_{i} x_{j}\right)_{i, j}$.

$$
\left\{\begin{array}{l}
\max \sum_{i=1}^{n} \alpha_{i}-\frac{1}{2} \sum_{i, j} \alpha_{i} \alpha_{j} y_{i} y_{j} \boldsymbol{x}_{i} \cdot \boldsymbol{x}_{j} \\
\forall i, \alpha_{i} \geq 0 \\
\sum_{i=1}^{n} \alpha_{i} y_{i}=0
\end{array}\right.
$$

We have the following property and decision function

$$
\boldsymbol{w}^{*}=\sum_{i=1}^{n} \alpha_{i}^{*} y_{i} \boldsymbol{x}_{i} \quad f(\boldsymbol{x})=\sum_{i=1}^{n} \alpha_{i}^{*} y_{i} \boldsymbol{x}_{i} \cdot \boldsymbol{x}+b
$$

Where $\alpha_{i}$ corresponds to the closest points not-null.

As error of treatment one introduces variables ' ressort' to soften the constraints, and one penalizes by the going beyond the constraint.

$$
\left\{\begin{array}{l}
\min \frac{1}{2}\|\boldsymbol{w}\|^{2}+C \sum_{i=1}^{n} \xi_{i} \\
\forall i, y_{i}\left(\boldsymbol{w} \cdot \boldsymbol{x}_{i}+b\right) \geq 1-\xi_{i}
\end{array}\right.
$$

In the not-separable case the dual problem will have the same form, the only difference is the upper limit on $\alpha$. Instead of seeking a hyperplane in input data, we access to an intermediate space of representation of great dimension.

$$
\begin{aligned}
\Phi: & \mathbb{R}^{d} \rightarrow \mathcal{F} \\
& \boldsymbol{x} \mapsto \Phi(\boldsymbol{x})
\end{aligned} \quad\left\{\begin{array}{l}
\max \sum_{i=1}^{n} \alpha_{i}-\frac{1}{2} \sum_{i, j} \alpha_{i} \alpha_{j} y_{i} y_{j} \Phi\left(\boldsymbol{x}_{i}\right) \cdot \Phi\left(\boldsymbol{x}_{j}\right) \\
\forall i, 0 \leq \alpha_{i} \leq C \\
\sum_{i=1}^{n} \alpha_{i} y_{i}=0
\end{array}\right.
$$

And the solution is

$$
f(\boldsymbol{x})=\sum_{i=1}^{n} \alpha_{i}^{*} y_{i} \Phi\left(\boldsymbol{x}_{i}\right) \cdot \Phi(\boldsymbol{x})+b
$$

The problem and its solution depend only on the scalar products $\Phi(\boldsymbol{x}) \cdot \Phi\left(\boldsymbol{x}^{\prime}\right.$. Rather than to choose the non-linear transformation $\Phi: \mathcal{X} \rightarrow \mathcal{F}$, we choose a function $k: \mathcal{X} \times \mathcal{X} \rightarrow \mathbb{R}$ called kernel function witch represents a scalar product in intermediate space representation and the examples distribution in this space.

$$
k\left(\boldsymbol{x}, \boldsymbol{x}^{\prime}\right)=\Phi(\boldsymbol{x}) \cdot \Phi\left(\boldsymbol{x}^{\prime}\right)
$$

Several cores are used with the most frequent SVM methods. In our case we have used a polynomial kernel.

$$
k\left(\boldsymbol{x}, \boldsymbol{x}^{\prime}\right)=\left(\boldsymbol{x} \cdot \boldsymbol{x}^{\prime}\right)^{d} \text { ou }\left(c+\boldsymbol{x} \cdot \boldsymbol{x}^{\prime}\right)^{d}
$$

The result image of this stage of spectral classification will be refined by the application of the Graph Cuts.

\section{GRAPH CUTS APPROACH.}

Graph Cuts can be used to conveniently minimize energies often used in computer vision. In this section we give a quick summary [9].

\section{A. The minimized energy}

The main idea of a graph cuts is to bring back the problem of minimization of energy to a problem of minimal cut in a graph.

Graph Cuts finds the optimal solution to a binary problem. However when each pixel can be assigned to many labels, finding the solution can be computationally expensive. For the following type of energy, a series of graph cuts can be used to find a convenient local minimum:

$$
\begin{aligned}
E(x) & =E_{\text {data }}+E_{\text {smooth }} \\
& =\sum_{i=1}^{p} D_{i}\left(x_{i}\right)+\sum_{\substack{i=1 . . n \\
j \in N_{i}}} V_{i j}\left(x_{i}, x_{j}\right)
\end{aligned}
$$

Where $E_{\text {smooth }}$ is a piecewise smoothness term, $E_{\text {data }}$ is a data term, $P$ is the set of pixels in the image, $V\left(x_{i}, y_{j}\right)$ is a smoothness penalty function to impose a spatial consistency, $D\left(x_{i}\right)$ is a data penalty function and $\left(X_{p}\right) p=1 \ldots n$ is a vector of variables in finite space $L$ ( $L$ for label). Every pixel is associated with a variable $X_{i} . N_{i}$ designate the set of the neighbors of the pixel $i$, and the construction of graph is done as follows:

Each pixel $P_{i j}$ in the image corresponds to a node $V_{i j}$ in the graph. Two additional nodes are the source and the sink, respectively the object and the background. Each node (pixel) is linked to its neighbors by edges n-link (neighbor), with connectivity chosen, and whose capacities depend on differences in intensity. Each node (pixel) is also connected by edges t-link to the terminals (source and sinks) Fig. 2.

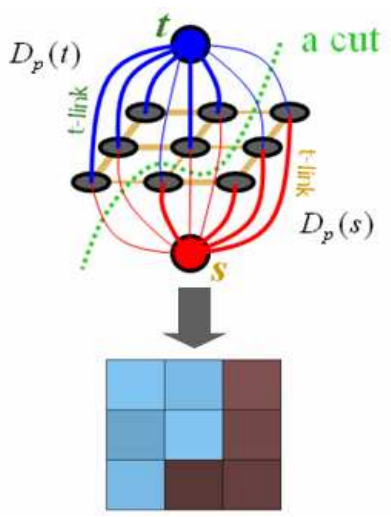

Figure 2. Construction of graph for a $3 \times 3$ image with the connectivity $V 8$. Each pixel corresponds to a node, and all pixel nodes are connected to the source and the sink.

A cut $C$ of graph $G(V, E)$ is a partition of the vertices $V$ into 
two sets, such that the terminals belong to each set. In combinatorial optimization, the cost of a cut is usually defined as the sum of the weights corresponding to the edges it serves [8] as is formulated in equation 11 .

$$
|C|=\sum_{i \in E} w_{i}
$$

In our case, one is going to use the algorithm alphaexpansion, this algorithm permits to minimize the energy $E$ on a finite set of labels $L$ and a class $V$ called penalty metric. $V$ is metric on the space labeled $L$ if it satisfies:

$$
\begin{gathered}
V(\alpha, \beta)=0 \Leftrightarrow \alpha=\beta \text { ои } V(\alpha, \beta) \neq 0 \Leftrightarrow \alpha \neq \beta \\
V(\alpha, \beta)=V(\beta, \alpha)>0 \\
V(\alpha, \beta)<=V(\alpha, \gamma)+V(\gamma, \beta)
\end{gathered}
$$

\section{B. Minimization algorithm}

The main idea of the alpha-expansion algorithm is to successively segment all $\alpha$ and non- $\alpha$ pixels with graph cuts and the algorithm will change the value of $\alpha$ at each iteration. The algorithm will iterate through each possible label for $\alpha$ until it converges.

At each iteration, the $\alpha$ region $P \alpha$ can only expand. This changes somehow the way to set the graph weights. Also when two neighboring nodes do not currently have the same label, an intermediate node is inserted and links are weighted so they are relative to the distance to the $\alpha$ label.
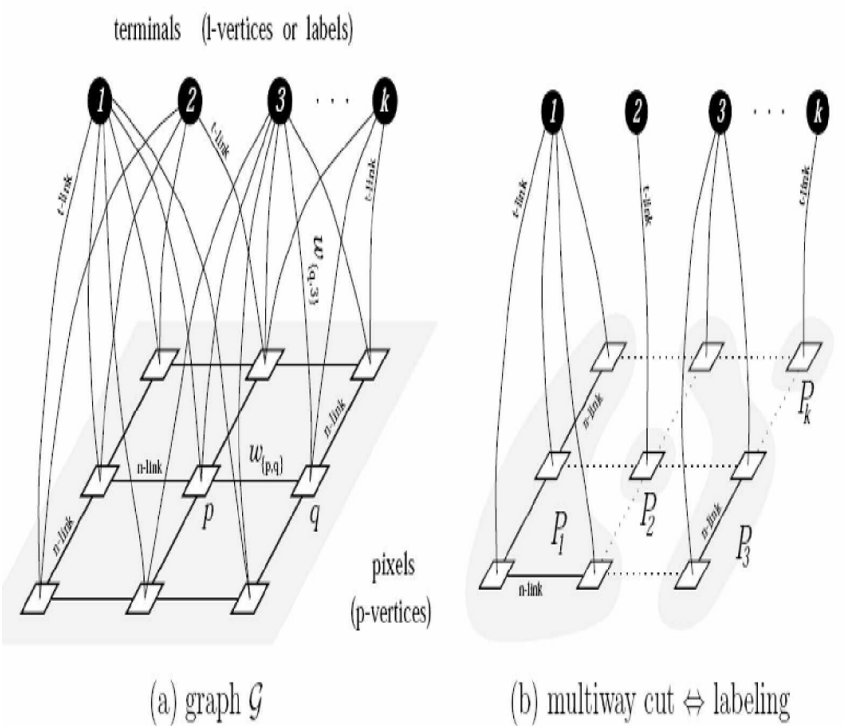

Figure 3. Generalization to a multi-objects. (a) A Graph $G=(V, E)$, with multiple terminals $1, \ldots \ldots, \mathrm{k}$ (b) A multi-way cut on $G$.

\section{EXPERIMENTATIONS AND RESULTS}

\section{A. Data}

We used two multispectral satellite images (Quickbird) represented in figure 4 (a), with respective size 240 by 360 pixels and 500 by 280 pixels. The idea for a good spectral classification of the pixels is to directly give the image's pixels value as input data of the classifier.

We have as at last, for each image, two files "TrainFile.dat" and "TestFile.dat" containing respectively 8516 and 8451 individuals (pixels) for learn, 86400 and 140000 individuals for test (to classify), and divided on the following classes:

\section{TABLE I. Different classes}

\begin{tabular}{cc}
\hline \hline Class N $^{\circ}$ & Class color \\
\hline 1 & Asphalte \\
2 & Green area \\
3 & Tree \\
4 & Soil \\
5 & Building \\
6 & Shadow \\
\hline \hline
\end{tabular}

\section{B. Results}

In this section we present the results obtained, first, with the SVM classifier. Thereafter, we show the interest of the use of the Graph Cuts to refine the result of the classification. We have used the toolbox "Support Vector Machine toolbox Version 2.51 (January 2002) of Anton Schwaighofer [6] (Institute for Theoretical Computer Science of Graz in Austria)" implemented in Matlab, with RBF kernel; but the results were not satisfactory, so we used SVM ${ }^{\text {light }}$ wich is an implementation of Support Vector Machines (SVMs) in C language [7] with RBF kernel.

To these images ((1-b) and (2-b) in Fig. 4) we have applied the morphological operations: opening and closing, what gives us the result images (1-c) and (2-c) in Fig. 4, and then we apply Graph cut approach to the same images what gives us the result images (1-d) and (2-d) that are represented in Fig 4.

At first sight, we note that we have a great difference between the results; the classification resulting from combining SVM-Graph cut is visually more satisfactory than those of SVM and SVM-Morphological operations since it is less noisy, while in the SVM classification we have an effect of salt and pepper. The resulting SVM-Graph Cut matches well with an urban land cover map in terms of smoothness of the classes, also it represents more connected classes. For the SVM-Graph Cut method a total accuracy of $96.05 \%$ was achieved, slightly higher than the accuracy of $92.45 \%$ derived by the SVM-Morphological operations classification and of $87.22 \%$ derived by the SVM classification (detailed in the following table)

TABLE II. TOTAL ACCURACY ACHIEVED BETWEEN TESTED IMAGES

\begin{tabular}{ccc}
\hline \hline Method & Image 1-a fig 4 & Image 2-a fig 4 \\
\hline SVM & $87.56 \%$ & $86.88 \%$ \\
SVM-Graph Cut & $96.13 \%$ & $95.97 \%$ \\
SVM-Morphological & $94.32 \%$ & $90.58 \%$ \\
operations & & \\
\hline \hline
\end{tabular}



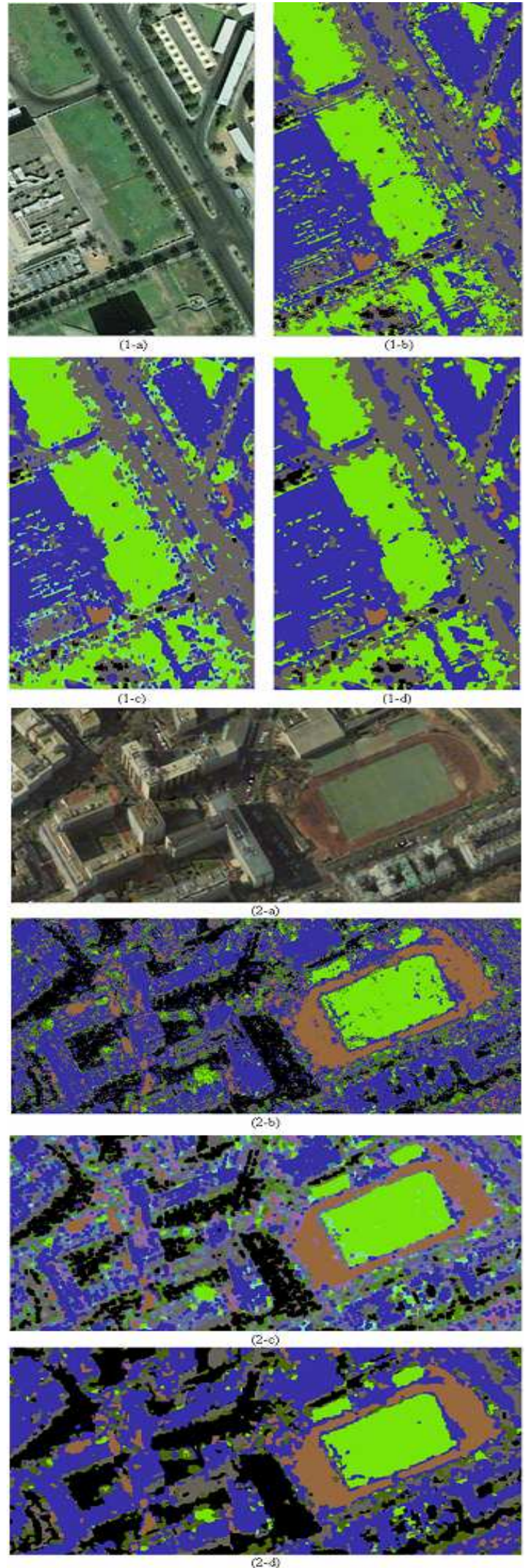

Figure 4. (1-a) and (2-a) original images, (1-b) and (2-b) result by SVM classification, (1-c) and (2-c) result by classifier SVM ameliorated by the morphological approach, (1-d) and (2-d) result by classifier SVM ameliorated by the graph cuts approach.

\section{CONCLUSION}

In this paper, we have presented a methodology which makes it possible to combine space and spectral information to refine the classification of multispectral satellite images. The application of this methodology gives more satisfactory results, however it remains to improve even more these results. In future work, to achieve this goal, we will be concentrating on the study of the cores in order to determine which is appropriate one for this type of classification.

This work was funded by CNRST Morocco and CNRS France Grant under "Convention CNRST CRNS" program.

\section{REFERENCES}

[1] Christophe SAMSON "Contribution à la classification des images satellitaires par approche variationnelle et équations aux dérivées partielles" : Thesis of doctorate, university of Nice-Sophia Antipolis.

[2] M. Fauvel, J. A. Benediktsson, J. Chanussot and J. R. Sveinsson "Spectral and Spatial Classification of Hyperspectral Data Using SVMs and Morphological Profiles" IEEE International Geoscience and Remote Sensing Symposium, IGARSS 07, July 2007, Barcelona Spain.

[3] Laetitia Chapel " Maintenir la viabilité ou la résilience d'un système : les machines à vecteurs de support pour rompre la malédiction de la dimensionnalité ? " : Thesis of doctorate, university of Blaise Pascal Clermont II.

[4] Sujeevan ASEERVATHAM " Apprentissage à base de Noyaux Sémantiques pour le Traitement de Données Textuelles ": Thesis of doctorate, university of Paris 13 -Galilée Institut Laboratory of Data processing of Paris Nord.

[5] Olivier Bousquet, " Introduction au Support Vector Machines (SVM) ", Center mathematics applied, polytechnique school of Palaiseau, 2001. http://www.math.u-psud.fr/ blanchard/gtsvm/index.html.

[6] Anton Schwaighofer "Support Vector Machine toolbox Version 2.51 (January 2002) Institute for Theoretical Computer Science of Graz in Austria. http://www.princeton.edu/ kung/ele571/571-MatLab/571svm/

[7] SVMlight Version: 6.02 Developed at University of Dortmund, Informatik, AI-Unit Collaborative Research Center on 'Complexity Reduction in Multivariate Data' (SFB475) . http://svmlight.joachims.org/

[8] Boykov, Y., Funka-Lea, G.: Graph Cuts and Efficient N-D Image Segmentation. International Journal of Computer Vision 70(2), 109-131 2006.

[9] Y. Boykov, O. Veksler et R. Zabih, Fast approximate energy minimization via graph cuts. IEEE International Conference on Computer Vision, volume 1, pages 377-384, septembre 1999. 